\title{
THE MAGNETIC AND VELOCITY FIELDS AND BRIGHTNESS IN THE SOLAR ATMOSPHERE
}

\author{
S. I. GOPASYUK and T. T. TSAP \\ Crimean Astrophysical Observatory, Nauchny, Crimea, U.S.S.R.
}

\begin{abstract}
Simultaneous observations of the magnetic fields, the line-of-sight velocities and brightness were made in active and quiet regions with the Crimean double-magnetograph in the following lines: $\mathrm{H} \alpha, \mathrm{K}_{3} \mathrm{CaII}, \mathrm{H} \beta, \mathrm{H} \gamma, \mathrm{H} \delta, \mathrm{MgI} \lambda 5184 \AA$, CaI $\lambda 4227 \AA, \mathrm{D}_{1} \mathrm{NaI}, \mathrm{BaII} \lambda 4554 \AA, \mathrm{CaI} \lambda 6103 \AA$, FeI $\lambda 5250 \AA$.

It is shown, that in the active regions the horizontal velocity is larger than the vertical one.

The mean velocities in the quiet solar photosphere have an isotropic distribution (Gopasyuk and Kalman, 1971).

The mean vertical velocities increase exponentially with height in active and quiet regions.

The correlation between velocities at different levels in active and quiet regions decreases with the distance between the levels of the formation of spectral lines, and it disappears for the velocities recorded in $\lambda 6103 \AA$ and $\mathrm{H} \beta$, for $\lambda 5184$ and $\mathrm{H} \alpha$ lines in active regions and for the velocities recorded in $\lambda 5250 \AA$ and $\mathrm{H} \alpha$ lines in quiet regions.

The position of the maximal field strength within a magnetic hill coincides statistically with the zero line of the line-of-sight velocities for active as well as for quiet regions.
\end{abstract}

Simultaneous observations of the magnetic fields, the line-of-sight velocities and brightness were made with the Crimean double-magnetograph (Severny, 1966). This magnetograph permits one to record magnetic fields, velocities and brightness in any two spectral lines simultaneously.

We used the following lines: $\mathrm{H} \alpha, \mathrm{K} 3 \mathrm{CaII}, \mathrm{H} \beta, \mathrm{H} \gamma, \mathrm{H} \delta, \operatorname{Mg}$ I $\lambda$ 5184, $\mathrm{CaI} \lambda 4227$, $\mathrm{D}_{1} \mathrm{NaI}, \mathrm{Ba} I \mathrm{I} \lambda 4554, \mathrm{Ca}$ I $\lambda$ 5103, Fe I $\lambda 5250$.

We have obtained many records in active and quiet regions at different distances from the center of the disk.

The entrance aperture was $2.8 \times 9.0$ and sometimes $2.7 \times 4.5 \mathrm{~s}$ of arc.

From maps of velocities we find the mean velocity for each region of outward and downward motions.

A comparison of these velocities for active regions at the center of the disk and near the limb shows that, in general, near the limb they are higher than at the center, which means that the horizontal motions have higher velocities than vertical ones. For example for the $\mathrm{H} \alpha$-line the horizontal component exceeds the vertical one by about a factor of two. At the same time, as was shown by Gopasyuk and Kalman (1971), the mean velocities in the quiet solar photosphere have an isotropic distribution (Figure 1).

The comparison of mean vertical velocities recorded in different lines at the disk center shows an increase of this velocity outwards with height for active regions as well as for quiet regions. Assuming that the spectral lines in active regions are formed at the same levels as in quiet regions, we find an exponential increase with the height.

$$
v(h)=V_{5250} \exp \left(h / h_{0}\right)
$$

where $h_{0}=3350 \mathrm{~km}$ and the level of the line formation $\lambda 5250$ is taken as zero. 
We also found that the vertical component of the mean velocity at the disk center (averaged over many active regions) is nearly equal to that obtained for the quiet Sun.

All said above is illustrated in Figure 2. The open circles show the downward motions and the crosses show the upward motions in active regions. The mean velocities in quiet regions are shown by solid dots. The solid line is the exponential curve which approximates the observations.

The correlation coefficients between velocities at different levels were computed for active and quiet regions at the disk center and we found that the correlation decreases with the distance between the levels of the formation of spectral lines. It practically disappears for the velocities recorded in $\mathrm{CaI} \lambda 6103$ and $\mathrm{H} \beta$ or for $\mathrm{MgI} \lambda 5184$ and

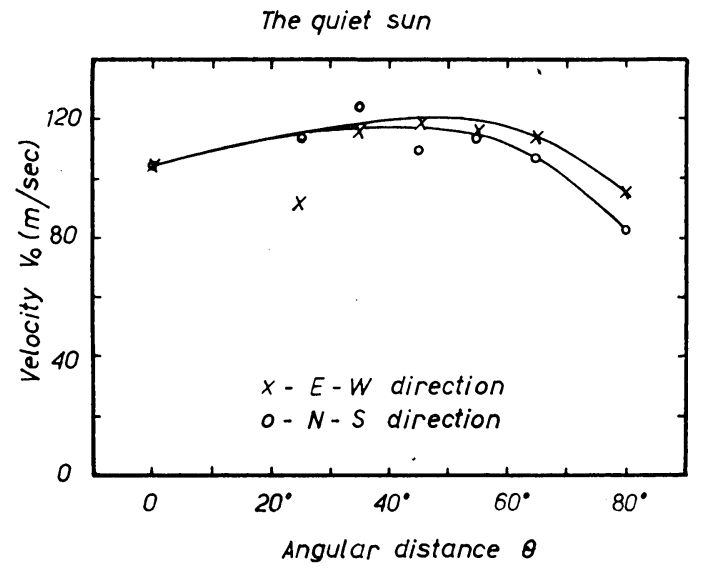

Fig. 1. The variation from center to limb of the mean line-of-sight velocity in the quiet solar photosphere. The records were made in the $\lambda 5250$ line.

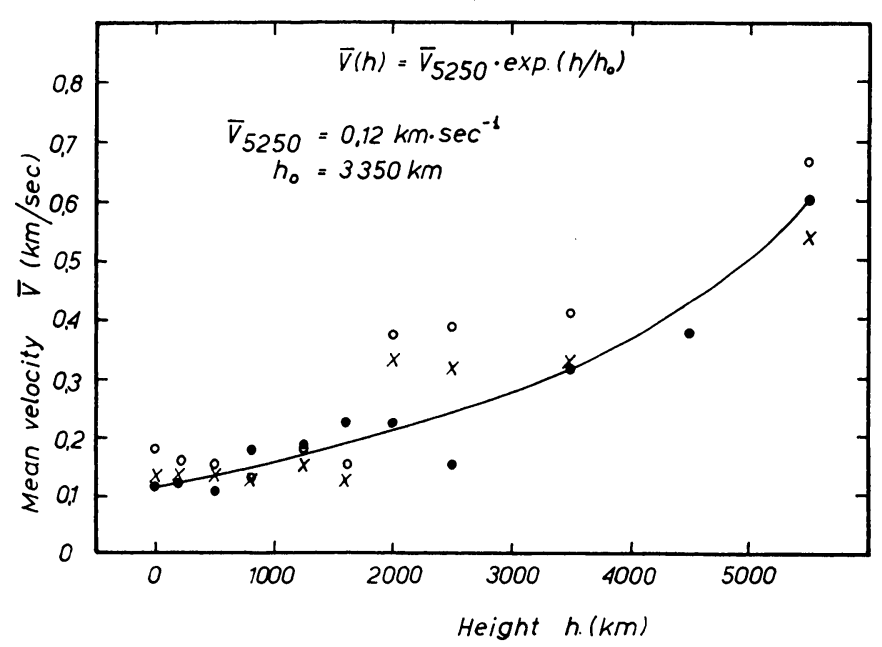

Fig. 2. The variation of the mean vertical velocities with height in active and quiet regions. 
$\mathrm{H} \alpha$ lines in the active regions and for the velocities recorded in $\mathrm{Fe} I \lambda 5250$ and the $\mathrm{H} \alpha$ line in quiet regions. In both cases of active and quiet regions the decrease of the correlation coefficients with distance between the levels can be described by the relation:

$$
R(h)=0.7-\left(\frac{h}{2 h_{0}}\right)^{2} .
$$

Here $h_{0}=2750 \mathrm{~km}$ for an active regions and $h_{0}=3350 \mathrm{~km}$ for a quiet region.

The variation of the correlation with distance in active and quiet regions is shown in Figure 3. The solid line corresponds to active regions and the dotted line to quiet regions.

Now, further we considered the correlation between the line-of-sight velocities and the longitudinal magnetic fields for active and quiet regions everywhere over the disk

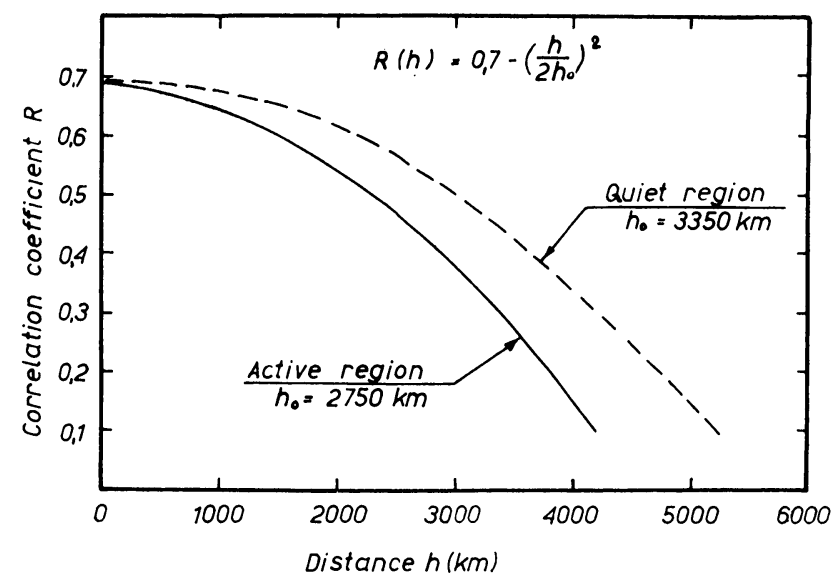

Fig. 3. The variation of the correlation with the distance between the velocities in active and quiet regions.

for different lines. The correlation coefficients for all lines except one corresponding to $\mathrm{K} 3 \mathrm{Ca}$ II in active regions do not exceed $0.10-0.15$. This leads probably to the conclusion that there is no correlation between line-of-sight velocities and longitudinal magnetic fields.

We have also found that the position of the maximum longitudinal field strength within a magnetic hill coincides statistically with the zero-line of the line-of-sight velocity for active as well as for quiet regions. This result follows from the measures of the shortest distances between the point of maximum field and the corresponding adjacent zero-line of line-of-sight velocity. Besides that we determine the distribution of the velocities in the points of maximum fields.

Both these results for quiet regions are shown in Figure 4. Figure 4 corresponds to the observations in the FeI $\lambda 5250$ line in quiet regions at the center of the disk. The upper curve shows the distribution of the shortest distances mentioned above. This is 
essentially a Gaussian distribution as can be seen from comparison of open circles with the solid line.

The curve below shows the distribution of the line-of-sight velocities at the points of the maximum field. The solid line is the exponential curve which approximates the observations.

In Figure 5 we have the same distributions found from the observations in the CaI $\lambda 6103$ line in active regions near the center of the disk $\left(\theta \leqslant 30^{\circ}\right)$.

The dispersion for the various distributions is $2600 \mathrm{~km}$ in the case of quiet regions and $3000 \mathrm{~km}$ for active regions.

We wish to emphasize that this kind of connection between the motions and magnetic

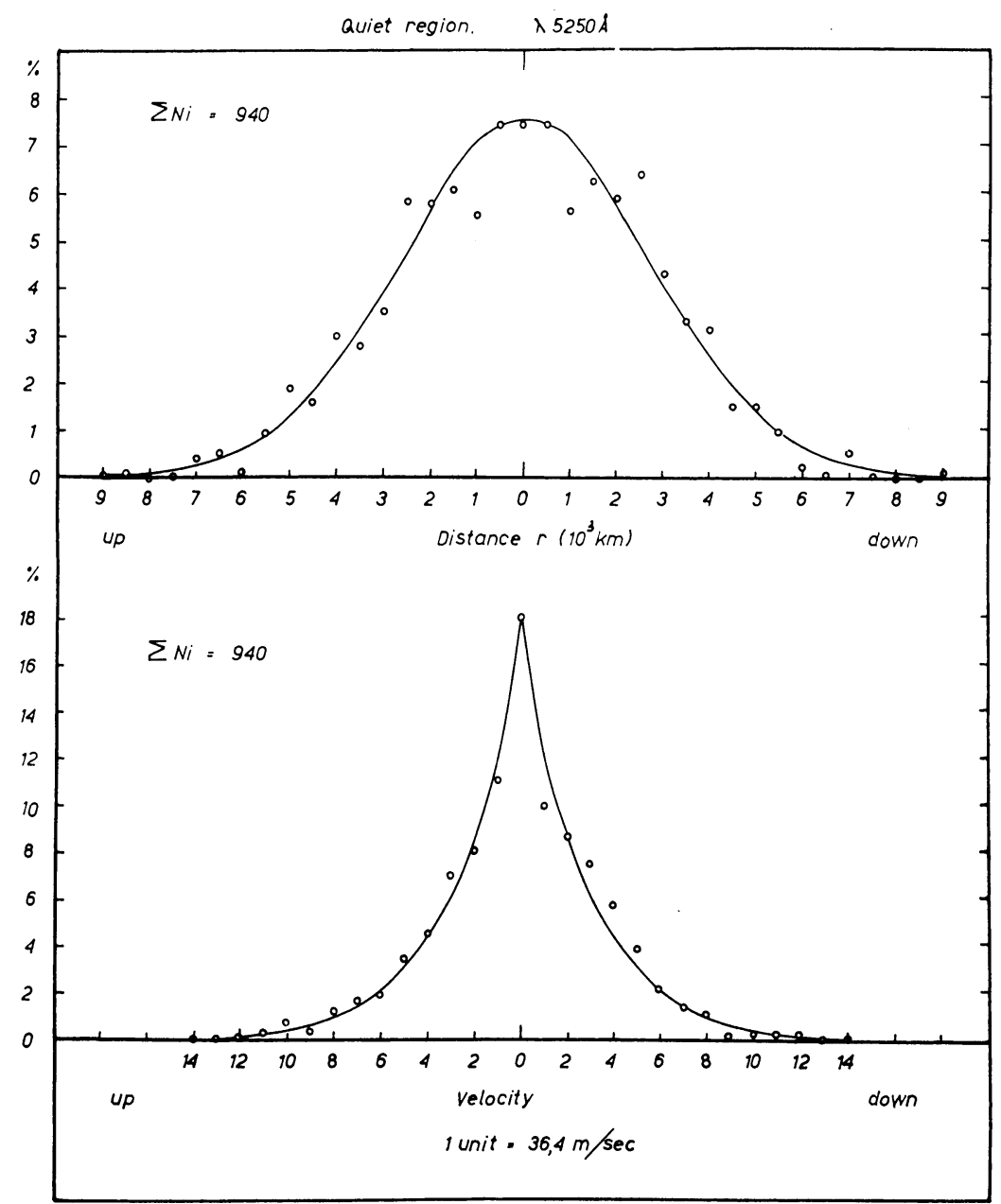

Fig. 4. The distribution of the shortest distances between the point of the maximum longitudinal field strength within a magnetic hill and the corresponding adjacent zero-line of line-of-sight velocity (the upper curve). The distribution of the line-of-sight velocities at the points of the maximum field (the lower curve) in the quiet regions at the center of the disk. 
fields remains the same at different levels in the solar atmosphere and at different distances from the center of the disk.

We should also point out that the maximum fields are usually positioned at the points of maximum brightness.

Finally we can conclude that (a) our observations are not in good agreement with

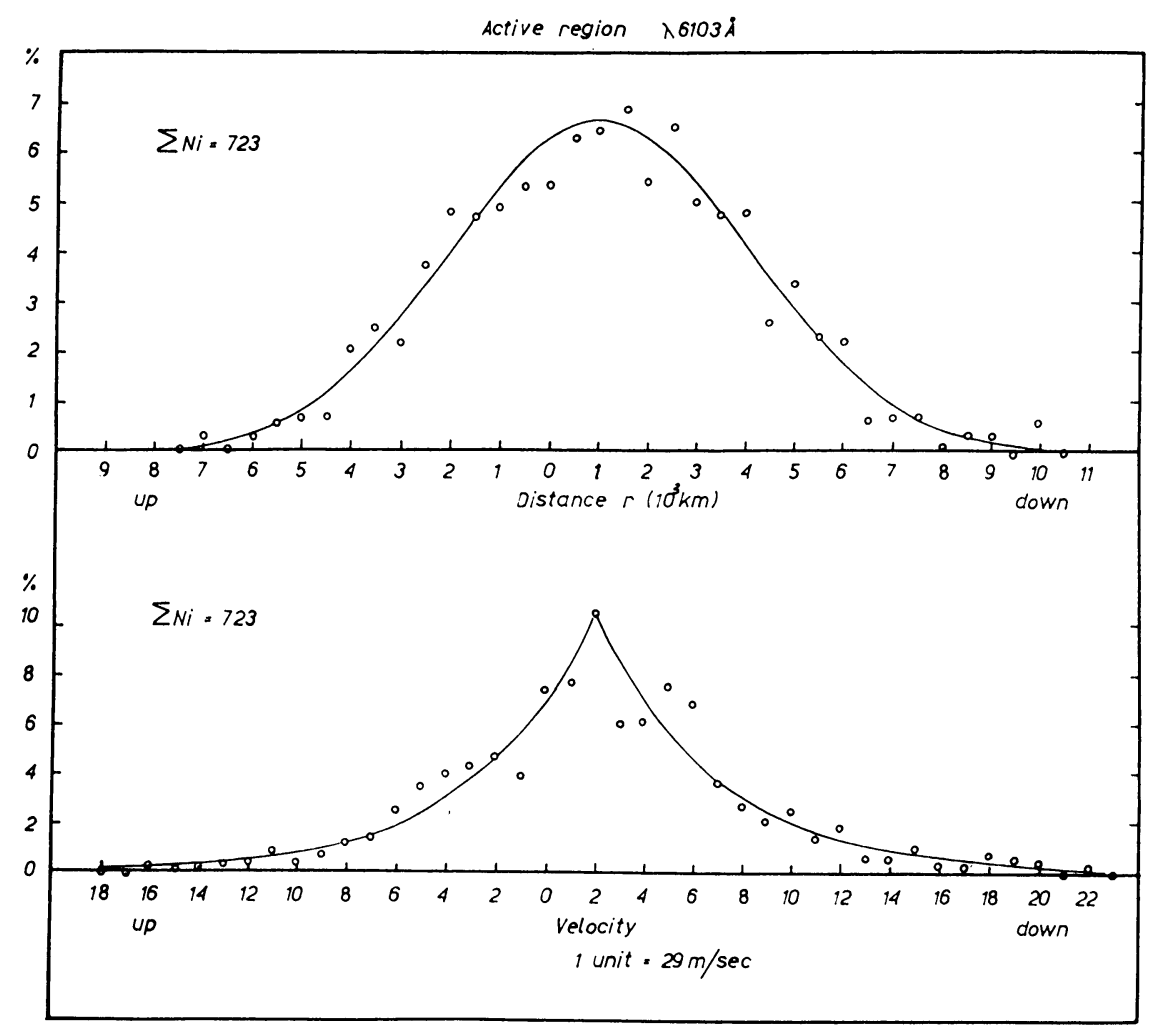

Fig. 5. The distribution of the shortest distances between the point of the maximum longitudinal field strength within a magnetic hill and the corresponding adjacent zero-line of line-of-sight velocity (the upper curve). The distribution of the line-of-sight velocities at the points of the maximum field (the lower curve) in active regions near the center of the disk.

the common picture of the super-granulation because they do not show predominant downward motions at the places of maximum field strength and brightness (b) our observations show that probably shock waves do not heat the chromosphere.

It seems to us that Ohmic losses may be important for the heating of the solar atmosphere.

\section{References}

Severny, A. B.: 1966, Astron. Zh. 43, 465.

Gopasyuk, S. I. and Kalman, B.: 1971, Izv. Krymsk. Astrofiz. Obs. 44, in press. 